\title{
Converting 2D-Medical Image Files “DICOM” into 3D- Models, based on Image Processing, and Analysing Their Results with Python Programming
}

\author{
RAFEEK MAMDOUH $^{1} \odot$, HAZEM M. EL-BAKRY ${ }^{2}$, ALAA RIAD $^{3}$, NASHAAT EL-KHAMISY $^{4}$ \\ ${ }^{1} \mathrm{PhD}$ candidate, faculty of Comp and Inf. System, Mansoura Univ. EGYPT, rafeekmty@hotmail.com \\ ${ }^{2,3}$ Information Systems Dept., Faculty of Computer \& Information Sciences, Mansoura University, \\ EGYPT \\ ${ }^{4}$ Computers and Information Systems Dept, Sadat Academy, Cairo, EGYPT \\ $\frac{{ }_{1}^{1} \text { rafeekmty@hotmail.com, }{ }^{2} \text { elbakry@mans.edu.eg, }{ }^{3} \text { amriad2014@gmail.com }}{{ }^{4} \text { wessasalsol@gmail.com }}$
}

\begin{abstract}
This paper presents the possibility of converting (2D) medical image data (Digital Imaging and Communications in Medicine (DICOM) files) to 3D model. Medical data and image processing software's, namely Seg3D2 and ImageVis3D, were used to analyze images, create 3D models of the liver and export them in OBJ images for performing a range of surgical procedures, and measure the accuracy of the size and weight of the liver, kidneys and arteries with their conformity to DICOM file. It is compared to the image processing before and after the conversion stage of medical image using the Python language program to ensure the integrity of the images after the conversion process is identical to the original pictures of DICOM without causing any distortions or changes to it. We reduce file size while maintaining the model's highest quality, while employing mixed reality techniques, applied on Liver Surgical Operation [living donor liver Transplantation (LDLT)].
\end{abstract}

Key-Words: Image Segmentation, 3D Meshes, 3D Processing, Medical Images, [DICOM], Image Processing Received: August 4, 2019. Revised: February 1, 2020. Accepted: February 21, 2020. Published: February 24, 2020.

\section{Introduction}

Medical files encompass patient's data, it is hard for the physicians to totally comprehend patient status using $2 \mathrm{~d}$ images. Thus, DICOM files must be transferred to $3 \mathrm{~d}$ imaging. The challenge will be discussed as follows:

In recent years, DICOM was recognized as a worldwide data innovation standard for acquiring electronic therapeutic pictures. Meta information data and pictures are combined in a single document with DCM extension. A solitary DICOM document sizes from MBs to GBs. The Picture Archiving Systems (PACS) utilizes a Relational Database Management System (RDBMS) to store and recover DICOM information. DICOM files stores huge patients' information. Extracting and recovering this huge information from substantial vaults is very unpredictable and testing. Applying enormous information methods for taking care of DICOM information will spares numerous patients' lives. Moreover, it enhances territories like research, treatment strategies, persistent likeness seeking, illness movement checking, clinical development, contextual analyses, preparing and learning, ability sharing and comprehends diverse examples in the medicinal picture information file in a secure way.
This paper presents a broad review of enormous specific information advances for DICOM information stockpiling and recovery and furthermore examinations, and the execution of Apache Pig, Hive

and Start while performing stockpiling and recovery of DICOM information.[1]

\section{Problem Definition}

First we will descript the problem in some words in the treatment of medical images, appearance of impurities / noise during processing and conversion of medical images files called (DICOM) .[2] [3] to $3 \mathrm{D}$ model where they are identical volume of the human organ according to the age and health status and empty from noises, to help the doctor / surgeon to interact with the disease through the (3D) model and the (liver) can be used as a case study .

To solve this problem we have to go through several steps using many different tools: 2D to $3 \mathrm{~d}$ converter, model, segment calculations and compare between images before and after :

1- Import/DICOM Files, de fine area and remove noises.

2- Compare between the two images before and after conversion. 
3- Determined segmentations and division "Masks, points and lines".

4- Calculate 3D model polygons and segmentations.

5- Enhance 3D model with edit segmants, Resculpting \& Triming.

6- Convert the OBJ Model to Low Poly Object through vertex editing, edge smoothing and sculpting, then, exporting to low poly object.

We will later discuss in detail solving the elements of the problem with illustration.

\section{Background}

\subsection{DICOM Overview}

DICOM [3] [4] in Fig.1, is a data processing, information storage and printing technique for medical imaging. It coordinates network communication and exchange of patient images and data through a communication protocol, an application that uses TCP / IP for inter-system communication. It integrates medical imaging devices such as servers, WS, scanner, network communications, image archiving systems, and communications. DICOM is widely used by hospitals and smaller practices such as dentists' offices and doctors.

Dicom is a set of images in the form of layers that are generally integrated via a special system to show the results of the combined rays clearly, as shown in in Fig. 2 herebelow.

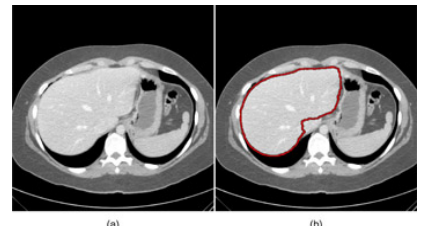

Fig. 1 Digital Imaging and Communication in Medicine (DICOM)

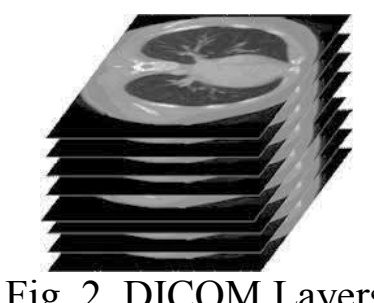

Fig. 2 DICOM Layers

Matrix processing, is a rising innovation to take care of the escallating demand on $f$ iguring force and capacity limit in fields, for example, atmosphere displaying, high-vitality material science, and lately life sciences. A protected, solid, and exceptionally effective information transport assumes a fundamental part in such lattice situations and much more so in medical networks. Lamentably, numerous lattice middleware conveyances, for example, the outstanding Globus Toolbox, do not have the joining of the overall restorative picture correspondence standard Advanced Imaging and Correspondence in Prescription (DICOM). As of now, the DICOM convention first should be changed over to the document exchange convention File Transfer Protocol (FTP) that is offered by the network middleware. [5]

\subsection{DICOM Standard Using}

DICOM gives itemized building data that can be utilized as a p art of interface details to empower organize availability among an assortment of items. The Standard depicts how to organize and trade medicinal pictures and related data, both inside the doctor's facility and outside the healing center (e.g., teleradiology, telemedicine). DICOM interfaces are accessible for association of any blend of the accompanying classifications of computerized imaging gadgets: (1) picture obtaining hardware (e.g., Fig. 2 tomography, attractive reverberation imaging, registered radiography, ultrasonography, and atomic pharmaceutical scanners); 2) picture documents; (3) picture preparing gadgets and picture show workstations; (4) printed version yield gadgets (e.g., photographic straightforwardness film and paper printers). DICOM is a message standard (i.e., data exchange between PC frameworks). DICOM is a far-reaching particular of data content, structure, encoding, and correspondences conventions for the electronic exchange of indicative and restorative pictures and picture related data. Some other medicinal services information trade gauges indicate just subgroup of the properties that affect interoperability. [6]

\subsection{BIG DATA}

BIG DATA [1] is an accumulation of tremendous measure of information (organized or semi-organized or unstructured) on the planet archives. Huge information creates an incentive from the store and preparing for bigger amounts of computerized information data that can't be broken down with conventional processing procedures [7] . Huge information isn't simply numbers, dates, and strings. Huge information is containing geospatial information, 3D information, medicinal picture information, sound, video, unstructured content, including $\log$ documents and online networking. Conventional information base frameworks are 
utilized for littler volumes of organized information, fewer updates or an anticipated, steady information structure. [8]. Enormous information investigation incorporates distinctive kinds of information. So the huge information innovations conquer the conventional framework impediment.

The Enormous information [9] qualities are volume, assortment, and speed. Huge information infers gigantic volumes of information. The volume of information is created by various asset frameworks like online networking the volume of information to be dissected is enormous. A huge amount of information refers to several sources of data. The information comes as $\mathrm{m}$ essages, photographs, recordings, observing gadgets, PDFs, sound, DICOM documents, and so forth. This Huge information assortment of unstructured information makes issues for capacity, mining and examining information. The stream of information is enormous and consistent, so it is exceptionally hard to deal with DICOM information.

The continuous [10] enormous information isn't only a procedure for putting away petabytes or Exabyte's of information in an information stockroom, it's about the capacity to settle on better choice and take on significant activities at the correct time. Quick forward to the advancements, like Hadoop, gives you the scale and adaptability to store information before we know how we will process it. Enormous information advancements, for example, Map reduce, HDFS, Tempest, Hive, Start, heron and so forth empower you to run questions without changing the information structures underneath.

[11] [12] Rossi and group exhibited that the therapeutic field contains everyone of the $3 \mathrm{Vs}$ of huge information; with roughly 500 pe tabytes of recuperatory tip generate through 2012 having an anticipated development of 25,000 petabytes by 2020 , it has an assortment with the dissimilar modalities (e.g. transcriptions, EEG, CT, X-Ray, DTI, PET, SPECT, MRS, fMRI, and so forth.) and it has speed in the quickness at which these modalities are made. Subsequently, it is basic to utilize enormous information advancements to make new human services applications that can devour this huge and shifting information.

Because of the attributes of restorative information applications [13], for example, the heterogeneity, the enormous/consistently expanding size, and the costly stockpiling, it is useful to misuse the energy of cloud-based frameworks, as Map Reduce, or its open source form Hardtop, Amazon Simple DB, Amazon Dynamo DB, Amazon RDS, SQL Sky blue, Pig, Hive to deal with such difficulties. This is on the grounds that these frameworks give promising arrangements of cost-adequacy, catastrophe recoverability, versatility, reasonability, and accessibility. By and by, none of these frameworks consider the multifaceted nature of the DICOM design.

\section{Related Works}

Eva Hnatkova, Petr Kratky and Zdenek Dvorak [2], Present a process to Convert medical data from $2 \mathrm{D}$ to $3 \mathrm{D}$ using one of the advanced conversion programs called Mimics and re-edit them until they are ready to print three-dimensional images, identical to human body anatomy. Yet, the tool used in the conversion does not fit with all cases of DICOM images.

M. Usman Akram, Aasia Khanum and Khalid Iqba [14], Provides a method for improving and dividing the liver using CT imaging by a global threshold and a set of equations to improve the liver image in terms of clarity and noise removal, while maintaining the size, density and shape of the liver. Yet, it is not easy to use the equations constantly, the image is almost accurate and requiers the intervention of some tools for more accurate drawing of equations.

Hongjian Shi and Rachid Fahmi [15], a study of liver tissue deformities using the current limit of F.E. by using high-resolution images of CBCT. Analysis of distortion rates through different linear models, and test of image distortion rates compared to simulation distortions. He explained that the flex line model is the best way to calculate the proportion of distortions and weights.

Van Sinh Nguyen, Manh Ha Tran, Hoang Minh Quang Vu [16], Presents a survey of The DICOM digital image conversion, provides a threedimensional model of digital and cross-sectional imaging and the use of the VTK library to analyze and construct the three-dimensional model.

Laura Mazzotta, Mauro Cozzani, Armando Razionale, Sabrina Mutinelli, Attilio Castaldo, and Armando Silvestrini-Biavati,[17] Present a survey of the formation of a three-dimensional model of dental roots without the use of CBCT, while reducing the radiation rate to maintain the health of the patient and provide three-dimensional information at the level of accuracy is acceptable statistically and clinically. However, the quality of the crown of the parametric model is weak, taking into account the various parameters (lengths of tendons, roots and widths) measured by $\mathrm{CBCT}$ and 
PAN. The cutters and bends were created during Bspline curves and free surfaces.

Himani S. Bhatt, Sima K. Gonsai, [18] In this research presents a fragmentation algorithm that was used to determine the appropriate area for conversion to a three-dimensional model using the "Cube Process" technique, an effective technique that provides doctors with a three-dimensional extension that includes a set of data that can be viewed from all angles

A. Farzana, Dr. M. Mohamed Sathik [19], this research presents the possibility of converting 2D DICOM MRI into 3D using one of the algorithms by relying on the matrices in the process of conversion, and the emergence of the results through the $\mathrm{K}$ Algorithem Technique, which is a compilation of algorithms as walking blocks, selecting the best results for acquiring a high rate of accuracy, privacy and sensitivity in information.

Padmaja Grandhe, Sreenivas Reddy Edara, Vasumathi Devara [20], presents in this research the use of low-resolution images through the technique of re-touch images, so as to improve the three-dimensional medical images and use a $3 \mathrm{D}$ Slicer-based imaging viewer in the graphical user interface.

Cam Q. T. Thanh, Nguyen T. Hai [21], the use of a technique to equate the histogram and the use of a multi-layer OTSU technique to divide each image to produce a fragment of the brain after a total of $2 \mathrm{D}$ magnetic resonance images have been treated to the cerebral cortex before converting it to a $3 \mathrm{D}$ image. Each part of the brain that is required to be seen from more From its angles, through the trilinear method, to construct a three-dimensional model of 2D-MRI.

In recent years, many researchers as shown in above have attempted to convert 3D files into three trigonometric methods using various algorithms, such as the use of algorithms and equations that can not be used in our case and conversion programs, including 3D Slicer and Mimics. However, there is sometimes a need for manual intervention in drawing and navigation and improvement of many images. Alternatively, we have used other conversion programs Seg3D2 and ImageVis3D through which you can see the images from three angles and convert them to a three-dimensional model, and then purify images and eliminate noises to obtain an integrated model, while maintaining the size and weight of the liver and its data as shown below in Table [ 1 \& 2].

\section{Method}

\subsection{Medical Image Process}

Increasing the light point [brightness and contrast] as show above Fig.3 with the number of light concentrated per radian area for image density improvement, when increase the light points will become density hence brighter images that are easy to interact conversion to $3 \mathrm{~d}$ model. Algorithm [1] and Fig. 4 presents python and open $\mathrm{CV}$ is an open source library, and specialize in data analysis, image processing and visualization, we used it to compare between medical image to insure it's empty from noise and ready to Convert to $3 \mathrm{~d}$ Model.

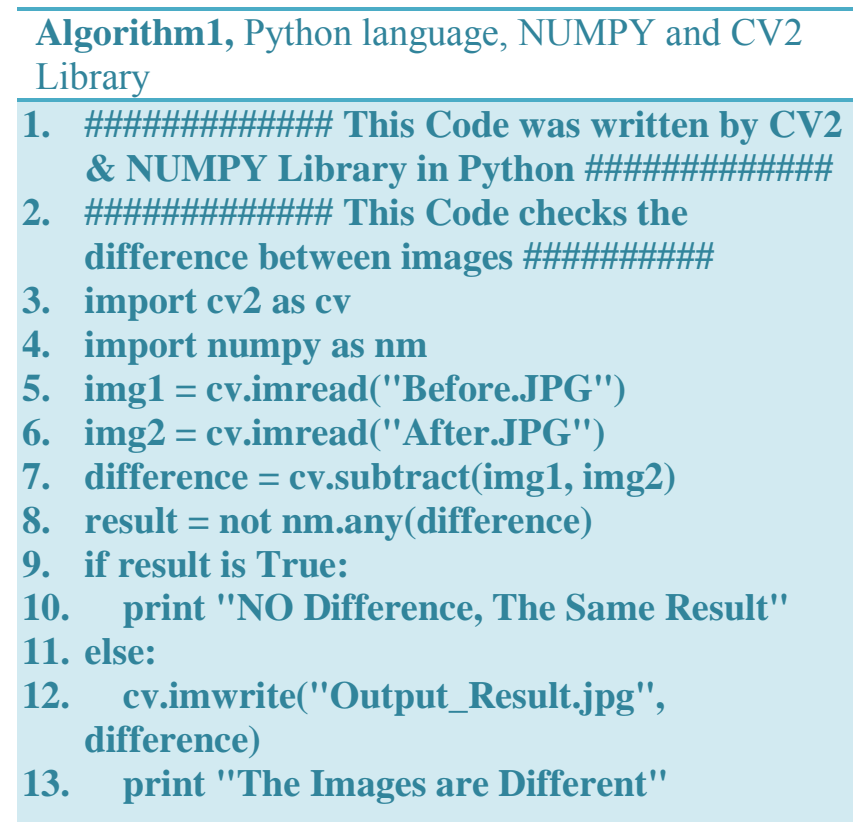

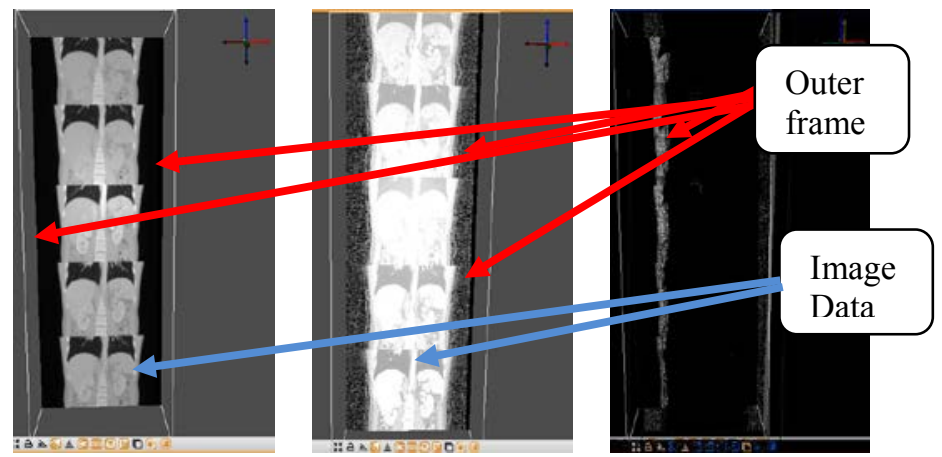

(C) $\begin{array}{ccc}\text { (A) Before } & \text { (B) After } & \begin{array}{c}\text { Comparative } \\ \text { Converting } \\ \text { Renverting }\end{array} \\ \text { Frame }\end{array}$

Fig. 3. Image Processing of DICOM File Before, After and Results 


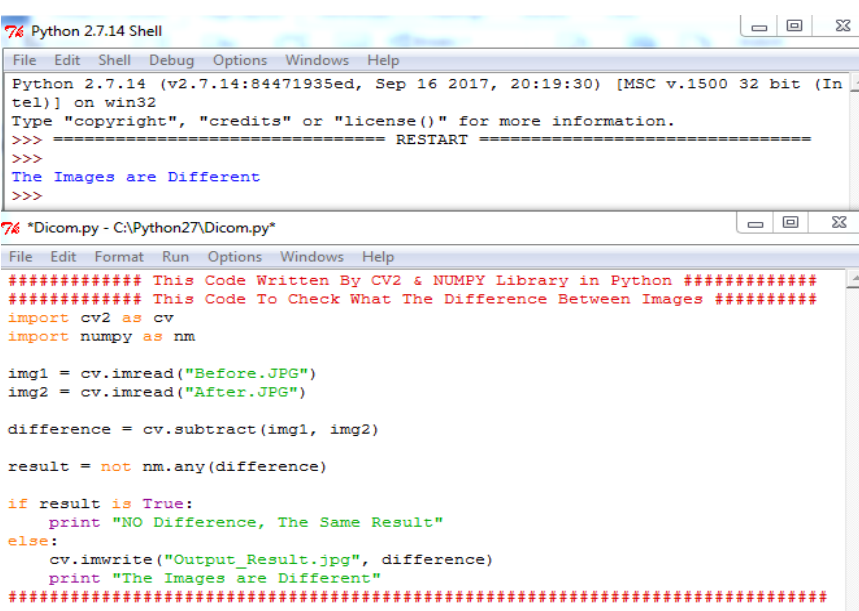

Fig. 4. Python Code Interface

\subsection{Convert To 3D Process}

The following flowchart is proposed to increase the performance of MR-based Liver Surgery Operation (LSO). This allows the integration of real physical objects using the three-dimensional models to enhance the work of the surgeon, by converting DICOM images to identical 3D models $\mathrm{X}+\mathrm{Y}+\mathrm{Z}=$ 1 , which will intersect at a confluence point to form the $3 \mathrm{D}$ model. Then, the resulting Model is inserted into the surgical training simulator before, during and after operations. Follow flowchart and PSEUDOCODE [Algorithm2] as shown below in Fig.5.

This section describes the architecture of the proposed model of DICOM conversion steps to a 3D model to enhance the liver surgery simulation (LSS) Algorithm [2]. Medical Simulation (MS) design based on mixed-reality technologies in user interface to promote (LSO) in three stages as described below. First and second phases analyze the DICOM files, remove noises, and converts it to a 3D file format. Third phase is result and testing convert DICOM files to 3D model low poly, which can be used with any virtual / simulation engine, follow Block Diagram Fig.6.
Algorithm2 : Converting DICOM File to3D Mode

1. Start Booting of computer.

2. Start Booting $\mathrm{S} / \mathrm{W}$

3. Input 2D Dicom (I/O)

4. Check Dicom Image $\operatorname{dim}=X, Y, Z$

5. if(Dicom $==X, Y, Z)$ is true Else Show Image Files on screen (I/O) end booting software ()

6. Check file Go to line 4

7. Convert Dicom Image : 3D Object

8. if(obj_file_dim=X,Y,Z) is true Export obj file HIGH POLY (I/O) Else Go To line 7()

9. Re_Touch 3D obj (Remove noise, Smooth, Segmant, Enhancement);

10. if(Re_Touch correct $=1)$ is true

11. Export obj file LOW POLY (I/O) Else Go To line 8()

12. Start Booting MR App

13. Insert low poly obj in Training App to check hardware performance

if(Training App is smoothing = 1) is true Render \& Display MR App

Else delay load process Go To line 9 Re_Touch \& Edit

14. End System 


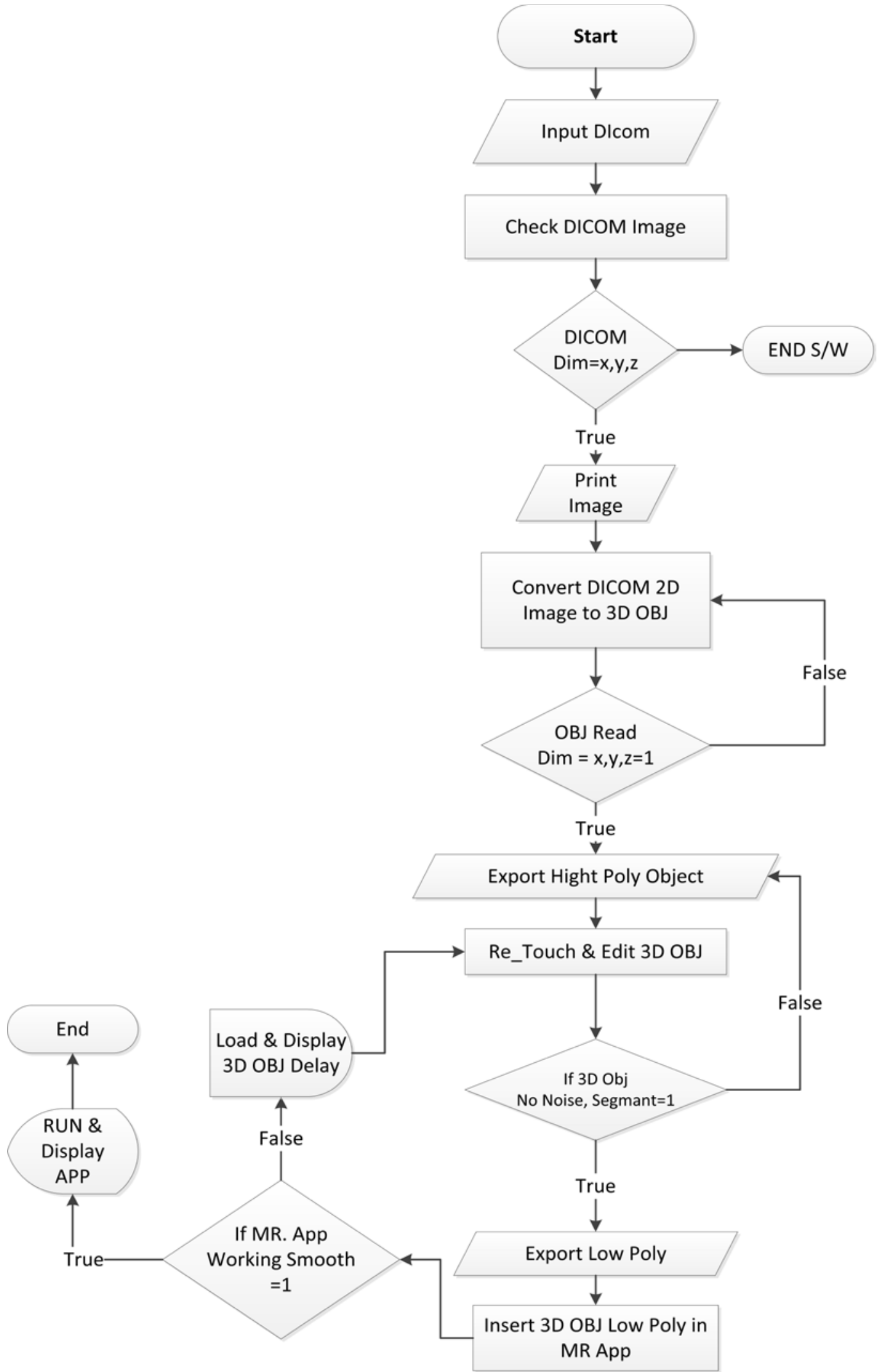

Fig.5 Flow Chart Converting DICOM File to3D Model 


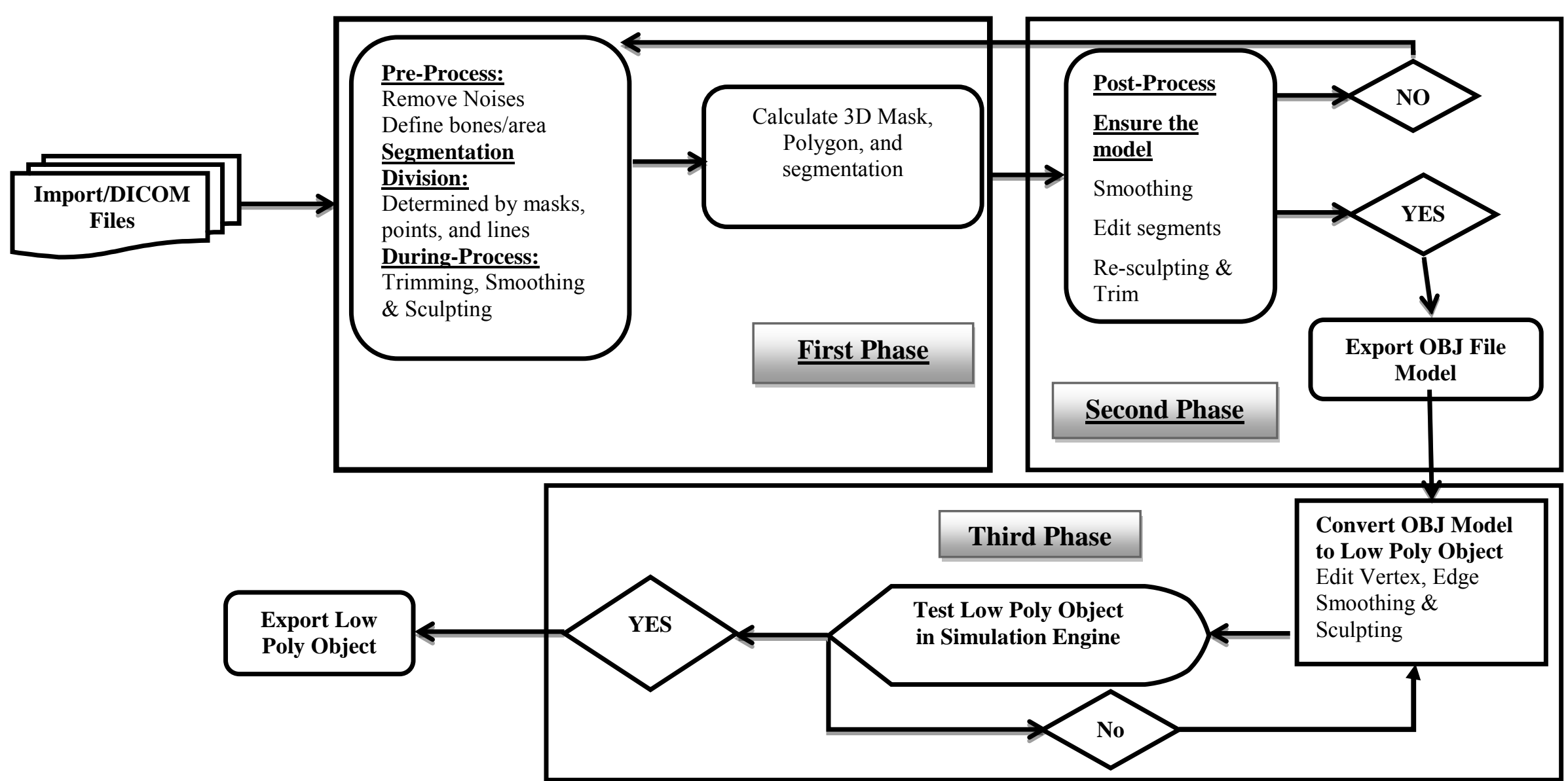

Fig. 6. Block Diagram of the Proposed Architecture for Converting Steps from DICOM to "3D Model / 3D object" Low Poly in Three Phase 
Table 1. Pre \& During Process Phase

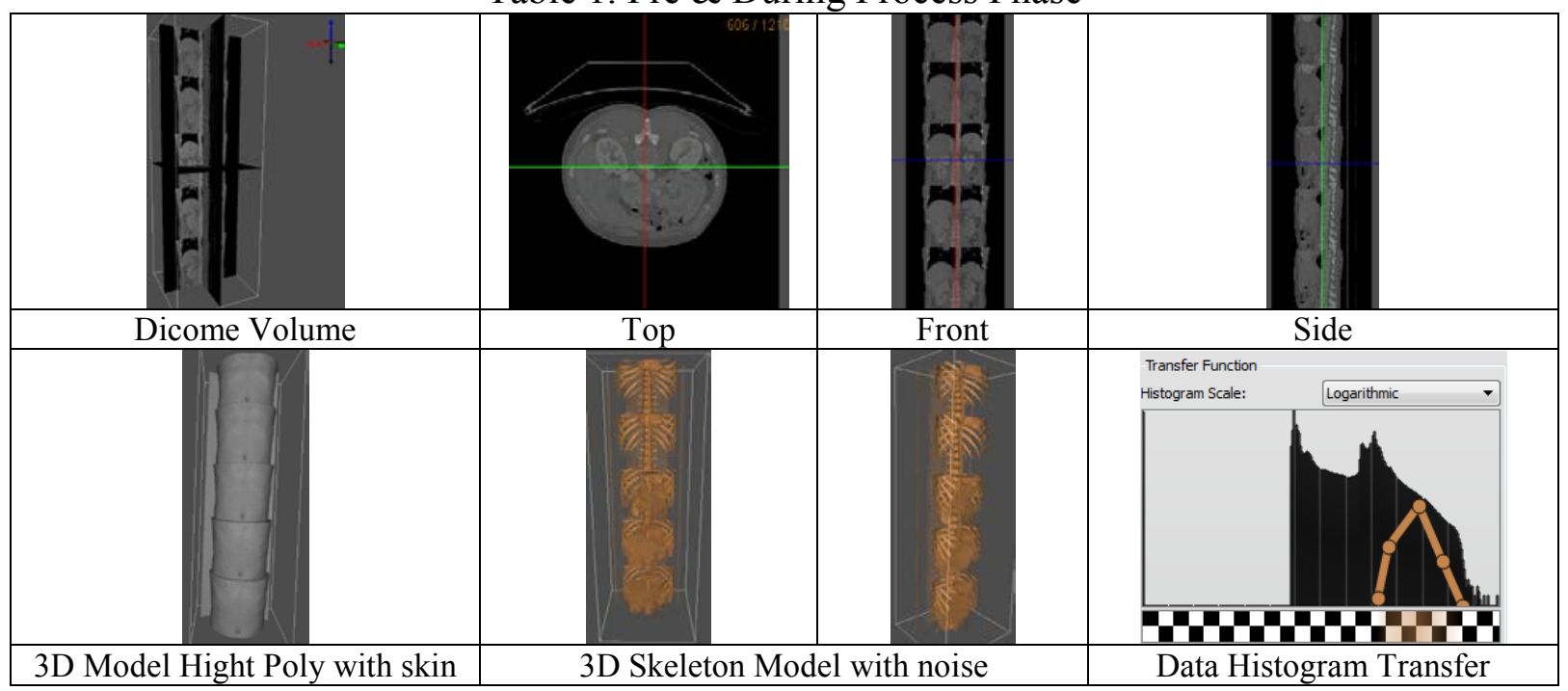

Table 2. Post-Process Phase

\begin{tabular}{|c|c|c|}
\hline \multicolumn{2}{|c|}{$\begin{array}{c}\text { Front \& Side OBJ File: [Before Edit segments, } \\
\text { Re-Sculpting \& Trimming] }\end{array}$} & $\begin{array}{c}\text { After Edit Segments, } \\
\text { Re-Sculpting \& Trimming }\end{array}$ \\
\hline & &
\end{tabular}

$1^{\text {st }}$ Pre \& During Process Phase: Start by converting files, they pass through several internal stages, import DICOM files, preprocessing to remove the noise, define the area to be modified by drawing by putting a set of points and lines with the execution of adjustments to determine the internal points required to show and deal with them. Trimming, smoothing and sculpting until we move to count the number of polygons and how to show them correctly and smoothly, in order to show all the details. Table 1 as shown above:

$2^{\text {nd }}$ Post-Process Phase: Make sure of the final form before exporting it. If it is identical to the required, it can be exported as OBJ file in two processes, see Before and After Edit Segments, Resculpting \& T riming Table 2 as shown above. The resulting $3 \mathrm{~d} \mathrm{M}$ odel can be used in a $3 \mathrm{~d}$ simulation engine. In case of nonconformity, go back to stage dead desired area and restore the same previous phase.

\section{Results Analysis}

This section discusses the results of our proposed model. The model is implemented using three different tools. The first tool is Autodesk 3D Maya, which is used to edit objects to low poly. While the second one is Autodesk Mesh mixer, which is used to define model to color parts and measure the stability volume and surface area. The third package called Python and CV2 is used to compare between $2 \mathrm{D}$ image before and after converting.

$3^{\text {rd }}$ Testing Phase: This is the most important phase through which the model will be tested in the final simulation and mixed with reality systems in which OBJ file is converted into a low poly model through the process of smoothing, sculpting and testing the extent of ease of movement within the graphics programs used, MESHMIXER. Thus, in case of total stability of the external perimeter and size of the mass of the liver and the kidney; the internal mass (Volume) can be estimated in $\mathrm{mm}^{3}$ and the external perimeter (Surface Area) in $\mathrm{mm}^{2}$., Fig.5. 
In order to match the files of the original DICOM with what is accessible, the file is converted to a 3D-model. The following steps shall be implemented:

1 - Open the DICOM file, made up of a group of images and improved brightness and contrast, so that we can convert them to a 3D-model.

2. Screen Shot obtained from the before and after files, Fig. 3(A), Fig. 3(B). Enhance clarification to ensure that there is no difference in the details of radiation.

3 - Assert the proper comparison between the two images using Algorithm [1], a Python language, NUMPY and CV2 Library, Fig. 4

\section{Results Evaluation}

- There is no difference in the basic details between the two pictures Fig. 3

- There is a difference only in the outer frame of the object Fig. 3(C) and it does not affect the process of conversion of the 3D-model, as it was deleted through the MESHMIXER

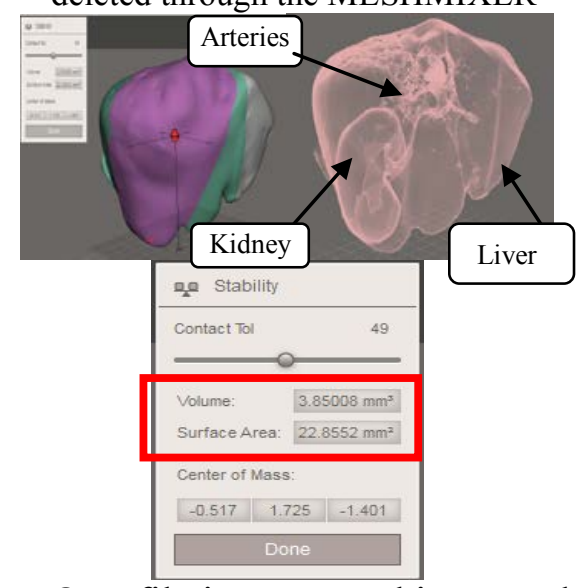

Fig. 7 : OBJ file is converted into an object low poly

\section{Conclusion and Future Work}

Overall, we can say that we succeeded in converting DICOM files, which is a set of serial images considered as a blueprint drawing, from 2D to 3Dmodel. DICOM files are considered as a medical database containing information about the patient's liver. The multi-programs provided by developers are imported to a simulation, and mixed with reality program, such as the Unity program, works easily during the render.

However, there are some active points still under study, as an attempt to convert 2D DICOM images to $3 \mathrm{D}$-model during X-rays, and to export them as an integrated model of all client data, that can be used directly within simulation and visualization (MR) systems.

\section{- Abbreviations:}

- LDLT: Living Donor Liver Transplantation

- DICOM: Imaging and Communications in Medicine

- MR: Mixed Reality

- PACS: Picture Archiving Systems

- RDBMS: Relational Database Management System

- FTP: File Transfer Protocol

- LSO: Liver Surgery Operation

- LSS: Liver Surgery Simulation

- MS: Medical Simulation

\section{Compliance with Ethical Standards:}

1- Funding: This study not funding

2- Conflict of Interest: No conflict exists: The authors declare that they have no conflict interests in this work.

\section{Authors' information:}

- Rafeek Mamdouh*'(1): Corresponding first author, Ph.D. candidate, Faculty of Computer and Information Systems, Mansoura University Egypt. Received BSC of (MIS) Management Information System in 2006, S horouk Academy, Cairo, Egypt, and M.Sc. in Computer Graphics and Information System, specializing in Visualization \& 3D Motion Capture, Sadat Academy 2013, Cairo, Egypt. Rafeek worked as an assistant lecturer at the British University, Shorouk Academy and other institutes from 2012 until 2018 and Multimedia and Graphics Dept., Faculty of Filmmaking \& Performing Arts, BADR University, Cairo, Egypt, 2018 until the present, rafeekmty@hotmail.com

- Prof. Dr. Hazem M. El-Bakry: 2nd author, Information Systems Dept., Faculty of Computer \& Information Sciences, Mansoura University, Egypt, elbakry@mans.edu.eg

- Prof. Dr. Alaa Riad: $3^{\text {rd }}$ author, Professor, and Ex-Dean of Faculty of Computers and Information Systems, Mansoura University, Egypt._amriad2014@gmail.com

- Prof. Dr. Nashaat El-Khamisy: $4^{\text {th }}$ author Prof. of Computers \& Information System, Dean of Sadat Academy for Management Sciences (SAMs), Cairo, Egypt, wessasalsol@gmail.com 


\section{References:}

[1] P. Haripriya and Dr. R. Porkodi, "An Efficient Storage and Retrieval of DICOM Objects using Big Data Technologies," International Journal of Advanced Research in Computer Science, pp. 271-274, 3 March 2017.

[2] Eva Hnatkova, Petr Kratky and Zdenek Dvorak , "Conversion of 2D medical scan data into 3D printed models," Advances in Environmental Sciences, Development and Chemistry, p. 315318, 2014.

[3] Wikipedia, "https://en.wikipedia.org/wiki/DICOM," 29 https://en.wikipedia.org/wiki/DICOM 2018. [Online]. Available: https://en.wikipedia.org/wiki/DICOM.

[4] DICOM and DICOMWeb, "Scope and Field of Application," in DICOM Part 1: Introduction and Overview , USA, the National Electrical Manufacturers Association, https://www.dicomstandard.org/current/, 2018, pp. 7-9.

[5] Thomas Tolxdorff, Michal Vossberg and Dagmar Krefting, "DICOM Image Communication in Globus-Based Medical Grids," IEEE Transactions on Information Technology in Biomedicine, pp. 145 - 153, 2008.

[6] W. Dean Bidgood, JR, Steven C. Horii, Fred W. Prior and Donald E. Van Syckle, "Understanding and Using DICOM, the Data Interchange Standard for Biomedical Imaging," Journal of the American Medical Informatics Association : JAMIA, p. 199-212., 1997.

[7] T.K.Das and P.Mohan Kumar, "Big Data Analytics: A Framework for Unstructured Data Analysis," International Journal of Engineering and Technology (IJET), 2013.

[8] E Dilsizian, Steven \& L Siegel and Eliot. , "Current Cardiology Reports," Artificial intelligence in medicine and cardiac imaging: harnessing big data and advanced computing to provide personalized medical diagnosis and treatment., no. 1534-3170, 13 Dec 2013.

[9] Kevin Normandeau , "Beyond Volume, Variety and Velocity is the Issue of Big Data Veracity," insideBIGDATA, Westborough, 2015.

[10] Ashwin Belle, Raghuram Thiagarajan, S. M. Reza Soroushmehr, Fatemeh Navidi, Daniel A. Beard and Kayvan Najarian, "BioMed Research International," Big Data Analytics in Healthcare, vol. 2015, p. 16, 16 June 2015.
[11] Hyejung Chang, Book Review: Data-Driven Healthcare \& Analytics in a Big Data World, Korean Society of Medical Informatics: Healthcare Informatics Research, 2015, p. 21(1): 61-62.

[12] Joachim Roski, George W. Bo-Linn and Timothy A. Andrews, "Health Affairs," Creating Value In Health Care Through Big Data: Opportunities And Policy Implications, pp. 1115-1120, july 2014.

[13] "Biomed Inform Insights," Big Data Application in Biomedical Research and Health Care: A Literature Review, pp. 1-10, 8 2016.

[14] M. Usman Akram, Aasia Khanum and Khalid Iqbal , " An automated System for Liver CT Enhancement and Segmentation," ICGST-GVIP Journal, vol. 10, no. IV, pp. 17-22, 2010.

[15] Hongjian Shi and Rachid Fahmi , "Energy Minimization within the F.E. Framework to Predict Liver Tissue Deformation," in GVIP 05 Conference, Cairo, Egypt, December 2005.

[16] Van Sinh Nguyen, Manh Ha Tran and Hoang Minh Quang Vu, "A Research on 3D Model Construction from 2D DICOM," in International Conference on Advanced Computing and Applications (ACOMP), Can Tho, Vietnam, 2016.

[17] LauraMazzotta, MauroCozzani, ArmandoRazionale, SabrinaMutinelli, AttilioCastaldo and andArmandoSilvestriniBiavati, "From 2D to 3D: Construction of a 3D Parametric Model for Detection of Dental Roots Shape and Position from a Panoramic Radiograph-A Preliminary Report," Hindaw iPublishing Corporation, International Journal of Dentistry , vol. 2013, 2013.

[18] Himani S. Bhatt and Sima K. Gonsai, "3D Modelling and Volume Rotation of 2D Medical Images," International Journal of Advanced Research in Electrical, Electronics and Instrumentation Engineering, vol. 3, no. 3, March 2014.

[19] A. Farzana and Dr. M. Mohamed Sathik , "Analysis of 2D to 3D Reconstruction Techniques over Brain MRI," International Journal of Innovative Research in Computer and Communication Engineering , vol. 5, no. 5, May 2017.

[20] Padmaja Grandhe, Sreenivas Reddy Edara and Vasumathi Devara, "Adaptive analysis \& reconstruction of 3D DICOM images using 
enhancement based SBIR algorithm over MRI," Biomedical Research, vol. 29, no. 4, pp. 644 - 653, 2018.

[21] Cam Q. T. Thanh and Nguyen T. Hai, "Trilinear Interpolation Algorithm for Reconstruction of 3D MRI Brain Image," American Journal of Signal Processing , vol. 7, no. 1, pp. 1-11, 2017. 